\title{
Psychiatric disorders in diabetes mellitus patients attending a tertiary health institution in north-eastern Nigeria
}

\author{
Kazeem Ayinde Ayanda, Dauda Sulyman, Mahmud Yinka Mahmud, Joy Pius, Simeon Yinka \\ Ategbese
}

Department of Psychiatry, Abubakar Tafawa Balewa University Teaching Hospital, Bauchi, Nigeria

\begin{abstract}
Psychiatric disorders are common sequelae of diabetes mellitus and can further worsen the clinical state and quality of life of patients. The aims of this study were to determine the prevalence and pattern of psychological illnesses in patients with diabetes mellitus and to assess factors that predict these psychiatric disorders. The mental health of 114 eligible consenting adult patients with diabetes mellitus attending the diabetic clinic of Abubakar Tafawa Balewa University Teaching Hospital was assessed using Mini International Neuropsychiatric Interview (MINI). The prevalence of psychiatric disorders in the study respondents was $39.5 \%$ and the pattern of diagnoses found were Major Depressive Disorder (31.6\%), Generalized Anxiety Disorder (6.1\%) and PostTraumatic Stress Disorder (1.8\%). None of the sociodemographic variables was significantly associated with the presence of psychiatric disorders. The presence of diabetic complications $(\mathrm{OR}=2.519 ; 95 \% \mathrm{CI}=1.056-6.001$; $\mathrm{P}$-value $=0.037)$ was predictive of psychiatric disorders while good sugar control $(\mathrm{OR}=0.372 ; 95 \%$ $\mathrm{CI}=0.162-0.854$; $\mathrm{P}$-value $=0.020)$ was a significant protective fac-
\end{abstract}

Correspondence: Ayanda Kazeem Ayinde, Department of Psychiatry, Abubakar Tafawa Balewa University Teaching Hospital, Bauchi, Nigeria.

Tel.: +234.8035065687

E-mail: kazeem12us@gmail.com

Key words: Diabetes mellitus, Psychiatric Disorders, Nigeria.

Contributions: all the authors contributed substantially to the design of the study and were actively involved in the acquisition of data. The data was analyzed and interpreted by AKA and the drafting and revision of the manuscript was done by both AKA and SD.

Conflict of interest: the authors declare no potential conflict of interest.

Funding: none.

Received for publication: 8 August 2018.

Revision received: 21 October 2018.

Accepted for publication: 27 October 2018.

This work is licensed under a Creative Commons Attribution NonCommercial 4.0 License (CC BY-NC 4.0).

(C) Copyright K.A. Ayanda, et al., 2018

Licensee PAGEPress, Italy

Annals of African Medical Research 2018; 1:41

doi:10.4081/aamr.2018.41 tor. A notable proportion of patients with diabetes mellitus develop mental health problems which can worsen the clinical outcome and further increase the burden of this illness. Efforts to promptly identify and manage these comorbid psychological problems can improve the prognosis of these patients.

\section{Introduction}

Diabetes Mellitus (DM) is a chronic metabolic disorder characterized by deficiency in insulin and/or insulin resistance with resultant elevation in blood glucose level. ${ }^{1}$ It is associated with a high level of disease burden particularly in developing world like Nigeria as it significantly impacts negatively on the finances, physical health and psychosocial functioning of the affected persons..$^{2,3}$ Worldwide, about 387 million people have diabetes and approximately $6 \%$ of them lives in the sub-Saharan region. ${ }^{4}$ About 4 million individuals live with this illness in Nigeria, however, majorities $(70-80 \%)$ are either unrecognized or untreated and most of them present late to health institutions with complications. ${ }^{4,5}$ Complications such as Diabetic ketoacidosis, nephropathy, retinopathy, neuropathy, erectile dysfunction are common at the time patients present to health facilities in Nigeria and these are responsible for the high level of economic burden attributed to diabetes mellitus. ${ }^{2,3,6}$

A diagnosis of DM poses extra challenges on patients and their caregivers as they are actively involved in diabetic management. DM patients are often required to follow strict dietary plans, engage in routine exercise, regularly monitor their blood glucose and adhere to drug treatment. ${ }^{7,8}$ In addition to the demand imposed by these lifestyle changes, patients also worry about the long term impart of diabetes on their physical, social and economic status and as such they may experience variety of emotional reactions like denial, anger, guilt feeling and depression at the time they are been screened or diagnosed with diabetes mellitus. ${ }^{9,10}$ Just like other chronic medical conditions, diabetes mellitus is associated with increased likelihood of serious psychological disorders such as depression, anxiety, eating disorders and schizophrenia, which have been reported by several researchers. ${ }^{7,11,12}$ Also documented in literature is the bi-directional association between DM and psychiatric disorders. ${ }^{12,13}$ Apart from the increased association of psychological problems in DM, there is a high associated risk of development of DM in patients with mental health problems. ${ }^{12-15}$ The co-morbid psychological problems in DM patients may interfere with patients' ability to care for themselves and to achieve good glycemic control. Thus, increasing the chances of developing diabetes complication, leading to rise in health care cost and hospital stay, as well as further impairing patients' quality of life and that of their caregivers. ${ }^{7}$

Most studies in Nigeria screened for the presence of psychological symptoms in DM patients with only few studies assessing 
for actual prevalence of psychiatric disorders and these few studies are more often restricted to anxiety and depressive disorders. With our extensive literature search, we did not find such study in the north-eastern region of this country. We aimed to determine the prevalence and type of mental health problems in patients with $\mathrm{DM}$ and to determine variables associated with these psychological problems. We hope that our findings may inform the need for physicians involved in DM care to pay attention to the psychological needs of their patients and appropriately refer patients to psychiatrists as early recognition and treatment of comorbid psychiatric problems would impact positively on patients' clinical outcome and improve the quality of life of both patients and caregivers.

\section{Materials and Methods}

This is a descriptive cross-sectional study carried out at the Diabetic Clinic of the medical out-patient department of Abubakar Tafawa Balewa University Teaching Hospital (ATBUTH) from September 2016 to February 2017. ATBUTH is a federal tertiary health institution located in the North-eastern region of Nigeria. The diabetic clinic is run every Wednesday by resident doctors and a Consultant Endocrinologist.

Only consenting adult patients with diagnosis of diabetes mellitus (DM) and on medication for at least 1 year were included in the study. Patients with comorbid chronic medical illness like hypertension, chronic kidney disease, patients with history of psychiatric illness prior to the diagnosis of DM and those who were too ill to participate were excluded from the study.

The ethics and research committee of ATBUTH gave approval for the study and permission to carry out the study was also obtained from the consultant endocrinologist in charge of the diabetic clinic. A total of 114 participants who presented consecutively and fulfilled the inclusion criteria over the 6-month period the study was undertaken were enrolled. A Pro forma questionnaire to obtain socio-demographic and clinical characteristics was designed and administered to eligible consenting participants. The socio-demographic variables include such factors as age, marital status, employment and educational status, while the clinical variables include factors such as the type of DM, duration of illness, family history of DM and presence and type of diabetic complications. Fasting blood sugar (FBS) is always done for all the patients routinely at every follow-up clinic. Patients were also required to record their FBS at scheduled intervals in between clinic appointments. Results of these FBS in patients' case-notes were used to determine those with good or poor sugar control.

All the respondents were administered Mini International Neuropsychiatric Interview (MINI 6.0) ${ }^{16}$ for assessment of psychiatric disorders by Consultant Psychiatrists. M.I.N.I is a short structured diagnostic interview for DSM-IV and ICD-10 psychiatric disorders. It was developed to meet the need for short but accurate structured psychiatric interview for multicenter clinical trials and epidemiology studies. M.I.N.I has an acceptably high validation and reliability scores and has been extensively used in the Nigeria settings. ${ }^{17}$

\section{Data analysis}

Data obtained from the study was analyzed using EPI-INFO version 6.04d (Centers for Disease Control and Prevention, CDC, Atlanta, Georgia, USA). Frequency tables were generated, and proportions were compared using chi square. The level of statisti- cal significant was set at 5\% confidence limit for 2-tailed test. Further analysis was done using logistic regression. Only variables that were statistically significant at the initial analysis were included in the logistic regression. This was done to determine variables that predict psychiatric morbidity among the studied population.

\section{Results}

\section{Sociodemographic characteristics of respondents}

One hundred and fourteen respondents were recruited over the 6-month study period, their age range was between 20 and 79 years (mean age $=50.86 \pm 12.54)$. Majority $(59.6 \%)$ of the respondents were responsible for the cost of their treatment (Table 1).

\section{Clinical characteristics of respondents}

Table 2 shows that majority of the study respondents (63.2\%) has a family history of diabetes mellitus, $101(88.6 \%)$ had type 2 diabetes mellitus (T2DM) and about $63.2 \%$ have good sugar control.

\section{Prevalence and types of psychiatric disorder in patients with diabetes mellitus}

Forty-five out of the 114 study respondents had psychiatric diagnoses, given a prevalence rate of $39.5 \%$ for psychiatric disorders. The prevalence rate of individual psychiatric diagnosis in the study population was Major Depressive Disorder (31.6\%), Generalized Anxiety Disorder (6.1\%), and Post-Traumatic Stress Disorder (1.8\%).

Table 1. Sociodemographic characteristics of respondents $(\mathrm{N}=114)$.

\begin{tabular}{lc}
\hline Variables & Frequency, n (\%) \\
Age group & \\
$\quad \leq 40$ years & $30(26.3)$ \\
$>40$ years & $84(73.7)$ \\
Sex & \\
$\quad$ Male & $43(37.7)$ \\
Female & $71(62.3)$ \\
\hline Religion & \\
Christianity & $32(28.1)$ \\
Islam & $82(71.9)$ \\
Marital Status & \\
Married & $95(83.3)$ \\
Never-Married & $19(16.7)$ \\
\hline Ethnicity & \\
Hausa/Fulani & $75(65.8)$ \\
Others & $39(34.2)$ \\
Employment Status & \\
Employed & $63(55.3)$ \\
Unemployed & $51(44.7)$ \\
\hline Educational status & \\
No Education/Primary & $58(50.9)$ \\
Secondary/Tertiary & $56(49.1)$ \\
Receiving Support & \\
Yes & $88(77.2)$ \\
No & $26(22.8)$ \\
\hline Source of Finance & $68(59.6)$ \\
Self & $46(40.4)$ \\
Other sources &
\end{tabular}




\section{Variables that predict psychiatric disorders}

Table 3 shows the comparison of sociodemographic factors between respondents with psychiatric disorders and those without psychiatric disorders. None of the variable in this category was found to be significantly associated with the presence of psychi-

Table 2. Clinical characteristics of respondents $(\mathrm{N}=114)$.

\begin{tabular}{lc}
\hline Variables & Frequency, n (\%) \\
Family history of DM & \\
Yes & $72(63.2)$ \\
No & $42(36.8)$ \\
Duration of Illness (years, mean= 7.85 \pm 6.25$)$ & \\
$<10$ & $78(68.4)$ \\
$\geq 10$ & $36(31.6)$ \\
\hline Type of DM & \\
Type 1 & $13(11.4)$ \\
Type 2 & $101(88.6)$ \\
Sugar Control & \\
Poor & $42(36.8)$ \\
Good & $72(63.2)$ \\
\hline Presence of Complication & \\
Yes & $36(31.6)$ \\
No & $78(68.4)$ \\
Types of Complications* & \\
Cerebrovascular accident (CVA) & $8(22.2)$ \\
CVA + Amputation & $1(2.8)$ \\
CVA + Retinopathy & $1(2.8)$ \\
CVA + Nephropathy & $1(2.8)$ \\
Amputation & $2(5.6)$ \\
Diabetic Retinopathy & $10(27.8)$ \\
Diabetic foot & $2(5.6)$ \\
Erectile Dysfunction & $9(25.0)$ \\
\hline Peripheral Neuropathy & $2(5.6)$ \\
Presence of Psychiatric Disorder & \\
Yes & $45(39.5)$ \\
No & $69(60.5)$ \\
\hline
\end{tabular}

${ }^{*}$ Total number of respondents with diabetic complications $=36$. atric disorder. However, in Table 4, longer duration of illness $\left(\chi^{2}\right.$ $=3.898 ; \mathrm{df}=1 ; \mathrm{p}$-value $=0.048)$, poor sugar $\operatorname{control}\left(\chi^{2}=8.690 ; \mathrm{df}=1\right.$; $\mathrm{P}$-value $=0.003)$ and the presence of complications $\left(\chi^{2}=7.833\right.$; $\mathrm{df}=1 ; \mathrm{P}$-value $=0.005)$ were clinical variables that were significantly associated with the presence of psychiatric disorders. These statistically significant variables were further subjected to a logistic regression analysis to determine factors that independently predicts the presence of psychiatric disorder among the study respondents. Both poor sugar control $(\mathrm{OR}=0.372 ; 95 \% \mathrm{CI}=0.162-0.854$; $\mathrm{P}$-value $=0.020)$ and presence of complication $(\mathrm{OR}=2.519 ; 95 \%$ $\mathrm{CI}=1.056-6.001 ; \mathrm{P}$-value $=0.037$ ) were found to predict psychiatric disorders in patients with Diabetes Mellitus (Table 5).

\section{Discussion}

The prevalence of psychiatric disorders in this study was $39.5 \%$ and the predominant diagnosis among our respondents was Major Depressive Disorder (MDD) with a prevalence rate of $31.6 \%$ followed by Generalized Anxiety Disorder (GAD) at $6.1 \%$, then Post-Traumatic Stress Disorder (PTSD) at $1.8 \%$. In a similar study among patients with Type 1 and type 2 diabetes mellitus using MINI International Neuropsychiatric Interview, Maia et al reported that $42.5 \%$ of their study participants have at least one psychiatric disorder and the pattern of disorders identified were GAD (21\%), dysthymia (15\%), social phobia (7\%), current depression $(5.5 \%)$, lifelong depression $(3.5 \%)$, panic disorder $(2.5 \%)$, and the risk of suicide $(2 \%) .{ }^{18}$ Many other researchers usually assess for the presence of depressive illness and anxiety disorder only, among diabetes mellitus (DM) patients unlike this study that investigated the whole range of psychiatric diagnoses. However, similar to findings in this study, many of them reported high prevalence rates for depressive disorders in patients with DM. ${ }^{19-22}$ The prevalence rate of depression in this study is comparable to those reported among DM patients in some Nigerian studies using diagnostic instruments. Igwe et al, using similar instrument as the one used in this study among their cohorts attending endocrinology

Table 3. Sociodemographic variables of the respondents with psychiatric disorders compared with those without psychiatric disorders.

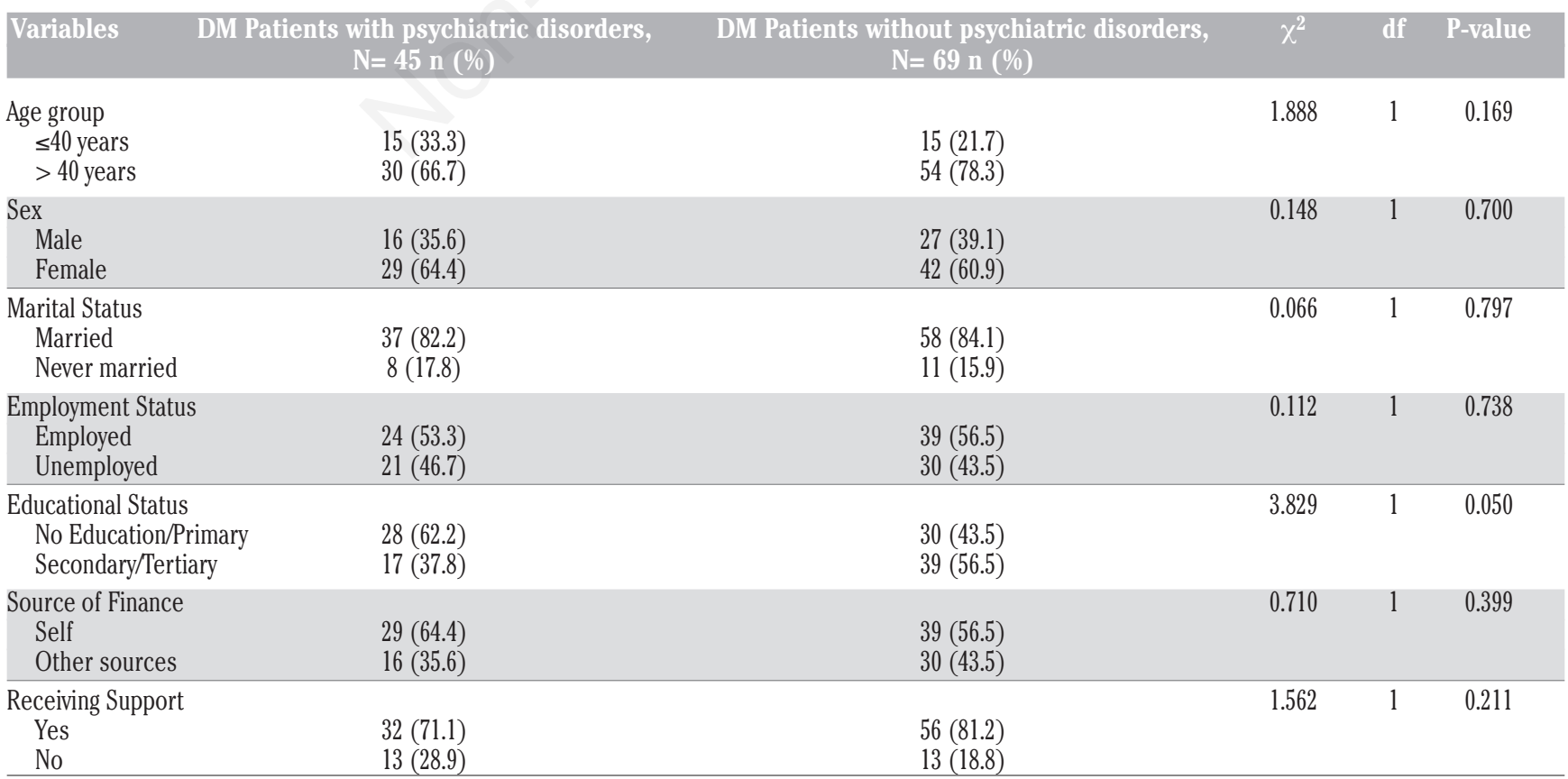


clinics of University of Nigeria Teaching Hospital, Enugu reported a prevalence rate of $27.8 \%$ among their subjects with DM. ${ }^{17}$ Likewise, James et al found a rate of $30 \%$ among their respondents with DM using the depression module of the Schedule for the Clinical Assessment in Neuropsychiatry (SCAN). ${ }^{19}$ However, the prevalence rate for depression in this study is much higher than the rate of $9.6 \%$ for depression reported among DM patients by Issa et al using Composite International Diagnostic Interview (CIDI), though the rate of $10.4 \%$ reported for anxiety disorder in their study is slightly higher than the rate in this study. ${ }^{23}$ Similarly, rates of $22.5 \%$ for MDD and $13.7 \%$ for GAD was found among DM patients in Bangladesh via a clinical interview using DSM-5 diagnostic criteria by Monzur et al. ${ }^{24}$ The higher proportion of females in this study compared to that by Issa et al and Monzur et al may be a plausible explanation for the higher rate of depression reported in this study as literature has revealed that females with DM are more likely to develop depression than their male counterparts. ${ }^{19}$

In other studies, using screening instruments, Gul \& Bali reported a much higher prevalence rates of $43 \%$ and $30 \%$ for depression and anxiety in DM patients attending outpatient clinic in India using Amritsar Depressive inventory (ADI) and Hamilton Anxiety Rating Scale (HARS) respectively. ${ }^{25}$ Likewise, the prevalence rate found in this study is lower than that reported in a community-based study among Type 2 diabetes mellitus (T2DM) patients in China using Zung-Self Rating Anxiety and Depression Scales where prevalence rates of $56.1 \%$ and $43.6 \%$ were found for depression and anxiety symptoms respectively. ${ }^{26} \mathrm{Also}$, Bahety et al. reported a rate of $63 \%$ for depression among T2DM patients in an out-patients clinic in Delhi, India using Patient Health Questionnaire (PHQ-9). ${ }^{20}$ The wide variation in prevalence rates of depression and anxiety disorders seen in our study and these other studies may be attributed to the differences in study settings, study populations and different instruments that were used. It is known that studies using screening instruments are more likely to over-diagnose depressive and anxiety disorders than studies where diagnostic instruments were used because some physical symptoms normally experienced by patients with chronic illnesses may be erroneously considered as psychiatric symptoms. ${ }^{13,27}$ It is important, therefore, to focus more on cognitive and behavioral symptoms of these psychiatric disorders. ${ }^{12}$ Conversely, in a Nigerian study by Coker et al using Wing's Present State Examination among DM patients attending an out-patient clinic, rates of $4 \%$ and $6 \%$ were reported for mild depressive and generalized anxiety disorders respectively. ${ }^{22}$ The prevalence rate for depression in their study was far below the rate reported by many other studies that used screening as well as diagnostic instruments. ${ }^{20,25,26}$ Apart from the fact that there is high proportion of males in their study, the study was carried out in a secondary health facility which may be attended by less severe cases of DM compared to tertiary health institution where this study was conducted.

None of the sociodemographic variables was significantly associated with the presence of psychiatric disorders in this study. It is of note that some Nigerian studies found socio-demographic variables such as been unmarried, lack of education, earning smaller income and low occupational status as factors that were significantly associated with psychiatric morbidity among patients with diabetes..$^{17,19,22}$ Also, in a Chinese study, female sex, old age, low education and being single were reported to be significantly associated with psychiatric symptomatology. ${ }^{26}$

In this study, presence of diabetic complications and poor sugar control were found to predict psychiatric disorders among our respondents. These findings are comparable to that by Bahety et al among T2DM attending endocrinology out-patients clinic in Northern India where occurrence of depression was significantly common among patients with diabetic complications and those with poor glycemic control. ${ }^{20}$ Likewise, El-Ela et al found among Egyptian patients with diabetes mellitus, that glycemic control was the most significant predictor of psychiatric morbidity. ${ }^{28}$ Similarly, in a community based cross-sectional study in Xuzhou, China among T2DM patients, diabetic complications were significantly associated with occurrence of depressive symptoms. ${ }^{26}$ It is important to note that the duration of illness in our study respondents was significantly associated with psychiatric morbidity in the initial analysis. This finding is similar to other

Table 4. Clinical characteristics of respondents with psychiatric disorders compared with those without psychiatric disorders.

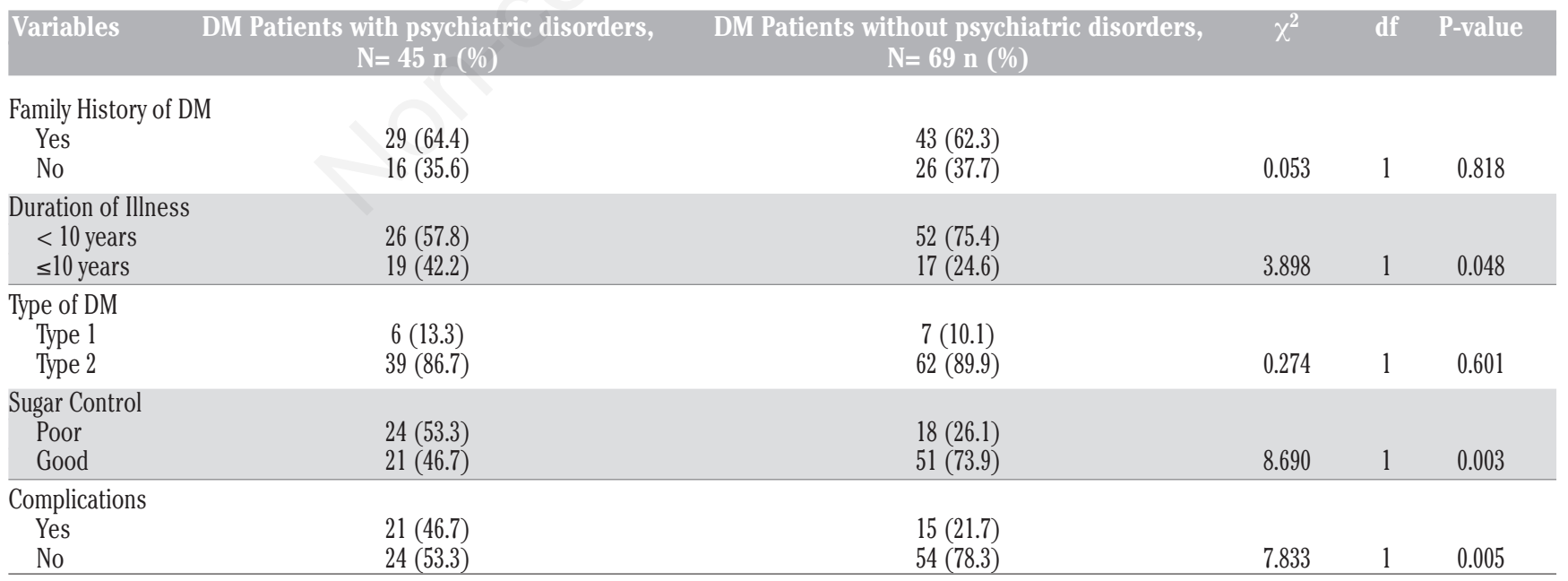

Table 5. Logistic regression of significant variables.

\begin{tabular}{lccccc} 
Variables & Odd Ratio & C.J. & Coefficient & Standard Error & P-value \\
Duration of Illness $(\geq 10 /<10)$ years & 1.477 & $0.610-3.571$ & 0.390 & 0.451 & 0.387 \\
Presence of Complication (Yes/No) & 2.519 & $1.056-6.001$ & 0.924 & 0.444 & 0.037 \\
\hline Sugar Control (Good/Poor) & 0.372 & $0.162-0.854$ & -0.990 & 0.424 & 0.020 \\
\hline
\end{tabular}


studies were illness duration was reported to be significantly associated with psychiatric morbidity among DM patients. ${ }^{20,22}$ However, this significance was lost in our study when duration of illness was subjected to logistic regression. This is in contrast to the finding by Sun et al, in which anxiety symptoms was significantly associated with longer duration of disease among T2DM patients in China. ${ }^{26}$

Diagnosis of DM is associated with lots of worries for the sufferers, which include but not limited to; concerns about the future in terms of the clinical outcomes, feeling of helplessness, lack of control over disease progression, and decision on disclosure to significant others especially their employers and insurers. ${ }^{7}$ These are coupled with the stress of having to follow strict dietary plan, exercise routines and blood sugar monitoring. Thus, the presence of complications and poor glycemic control obviously add to the overall burden of disease on these patients who are already overwhelmed by the lifestyle changes and socioeconomic consequences of living with the disease and as such increase their risk of developing psychological disorders. ${ }^{10}$ The presence of mental health problems consequently deepens DM patients' inability to take care of themselves, adhere to management plans and worsens their general wellbeing and expectedly those of their caregivers. The fact that this is a cross-sectional study and not a longitudinal one is a major drawback because the latter might be more reliable at recognizing factors that predispose to psychological disorders in chronic medical conditions like DM. The finding in this study may not be generalizable due to its small sample size. Future studies involving multiple centers and large sample sizes would possibly increase their generalizability.

\section{Conclusions}

These researchers concluded that diabetes mellitus is a chronic debilitating condition that is frequently complicated by psychiatric illnesses. A high rate of about $39.5 \%$ was found in this study. The presence of psychological disorders may impact negatively on the abilities of the sufferers to follow management plans, thereby worsening their clinical outcome and health satisfaction. It is therefore imperative for physicians caring for DM patients to improve their knowledge on mental health via continued medical education, to pay extra attention to the psychological needs of their patients, regularly screen patients for psychological symptoms and liaise with Psychiatrists in the care of those patients who develop mental health problem. These approaches may go a long way in improving the clinical outcome of patients with Diabetes mellitus.

\section{References}

1. Doherty AM. Psychiatric aspects of diabetes mellitus. Br J Psych Adv 2015;21:407-16.

2. Ogbera AO, Ekpebegh C. Diabetes mellitus in Nigeria: the past, present and future. Wrld J Diabetes 2014;5:905-11.

3. Kayode OO, Odukoya OO, Odeniyi IA, et al. Pattern of complications and comorbidities among diabetic patients in a tertiary healthcare center in Nigeria. J Clin Sci 2015;12:29-35.

4. Fasanmade OA, Dagogo-Jack S. Diabetes care in Nigeria. Ann Global Health 2015;81:821-9.

5. Dahiru T, Aliyu A, Shehu AU. A review of population-based studies on diabetes mellitus in Nigeria. Sub-Sahar Afr J Med 2016;3:59-64.

6. Oputa RN, Chinenye S. Diabetes in Nigeria: a translational medicine approach. Afr J Diabetes Med 2015;23:7-10.

7. Trigwell P, Peveler R. Psychiatric aspects of diabetes mellitus.
APT 1998;4:159-66.

8. Clarke J, Forde R. Psychosocial issues in diabetes. WIN 2006:434. Available from: https://www.inmo.ie/ MagazineArticle/PrintArticle/5736

9. Sridhar GR. Psychiatric co-morbidity \& diabetes. Indian J Med Res 2007;125:311-20.

10. Young EE, Unachukwu CN. Psychosocial aspects of diabetes mellitus. Afr J Diabetes Med 2012;20:5-7.

11. Taneja N, Adhikary M, Chandramouleeswaan S, Kapoor SK. Prevalence of common mental disorders among patients with diabetes mellitus and hypertension in an urban east Delhi slum-a Cross sectional study. TJP 2015;1:27-32.

12. Balhara YPS. Diabetes and psychiatric disorders. Indian J Endocrinol Metab 2011;15:274-83.

13. Pan A, Lucas M, Sun Q, et al. Bidirectional association between depression and type 2 diabetes mellitus in women. Arch Intern Med 2010;170:1884-91.

14. Medved V, Jovanovic N, Knapic VP. The comorbidity of diabetes mellitus and psychiatric disorders. Psychiat Danub 2009;21:5858.

15. Robinson DJ, Luthra M, Vallis M. Diabetes and mental health. Can J Diabetes 2013;37:s87-92.

16. Sheehan DV, Lecrubier Y, Hamett-Sheehan K, et al. The mini international neuropsychiatric interview (MINI): the development and validation of a structured diagnostic psychiatric interview. $\mathrm{J}$ Clin Psychiatry 1998;59:22-33.

17. Igwe MN, Uwakwe R, Ahanotu CA, et al. Factors associated with depression and suicide among patients with diabetes mellitus and essential hypertension in a Nigerian teaching hospital. Afr Health Sci 2013;13: 68-77.

18. Maia AC, Braga Ade A, Brouwers A, et al. Prevalence of psychiatric disorders in patients with diabetes 1 and 2. Compr Psychiatry 2012;53:1169-73.

19. James BO, Omoaregba JO, Eze G. Depression among patients with diabetes mellitus in a Nigerian teaching hospital. SAJP 2010;16:61-4.

20. Bahety P, Agarwal G, Khandelwal D, et al. Occurrence and predictors of depression and poor quality of life among patients with type-2 diabetes: a Northern India perspective. Indian J Endocrinol Metab 2017;21:564-9.

21. Ornelas ACC, Alves VM, Carta MG, et al. Mental disorders in subjects with diabetes: a systemic review. Health Care Curr Rev 2017;5:1-7.

22. Coker AO, Ohaeri JU, Lawal RA, Orija OB. Specific psychiatric morbidity among diabetics at a Nigerian general hospital. East Afr Med J 2000;77:42-5.

23. Issa BA, Yussuf AD, Baiyewu O. The association between psychiatric disorders and quality of life of patient with diabetes mellitus. Iran J Psychiatry 2007;2:30-4.

24. E-Monzur S, Taher A, Roy S, et al. Major depressive disorder and generalized anxiety disorder among the patients with diabetes mellitus. Bang J Psychiatry 2015;29:14-7.

25. Gul D, Bali K. A study of comorbidity of depression, anxiety and diabetes mellitus. IJMDS 2017;6:1513-7.

26. Sun N, Lou P, Shang Y, et al. Prevalence and determinants of depressive and anxiety symptoms in adult with type 2 diabetes in China: a cross-sectional study. BMJ Open 2016;6:1-8.

27. Goldberg D. the detection and treatment of depression in the physically ill. Wrld Psychiatry 2010;9:16-20.

28. El-Ela EA, Shorub E, Khalifa D, Ashour R. Psychiatric morbidity and quality of life among adults with diabetes mellitus. Middle East Curr Psychiatry 2015;22:132-6. 